\title{
Fluorine-Incorporated Amorphous Carbon Coating Inhibits Adhesion of Blood Cells to Biomaterials
}

\author{
Ayumi Horikawa, ${ }^{1,2 \dagger}$ Shunto Maegawa, ${ }^{3 \dagger}$ Terumitsu Hasebe, ${ }^{3,4 \dagger^{*}}$ Tomohiro Matsumoto,, 3 \\ Minoru Tanaka, ${ }^{2,5}$ Koki Takahashi, ${ }^{2,6}$ and Tetsuya Suzuki ${ }^{3}$ \\ ${ }^{1}$ Graduate School of Humanities and Science and Glycoscience Institute, Ochanomizu University, \\ 2-1-1 Otsuka, Bunkyo-ku, Tokyo 112-8610, Japan \\ ${ }^{2}$ Department of Transfusion Medicine and The Institute of Medical Science, the University of Tokyo, \\ 7-3-1 Hongo, Bunkyo-ku, Tokyo 113-8655, Japan \\ ${ }^{3}$ Center for Science of Environment, Resources and Energy, Graduate School of Science and Technology, \\ Keio University, 3-14-1 Hiyoshi, Kohoku-ku, Yokohama, Kanagawa 223-8522, Japan \\ ${ }^{4}$ Department of Radiology, Tokai University Hachioji Hospital, Tokai University School of Medicine, \\ 1838 Ishikawa-machi, Hachioji-shi, Tokyo 192-0032, Japan \\ ${ }^{5}$ Division of Innovative Cancer Therapy, The Advanced Clinical Research Center, \\ The Institute of Medical Science, the University of Tokyo, 7-3-1 Hongo, Bunkyo-ku, Tokyo 113-8655, Japan \\ ${ }^{6}$ Department of Blood Service Board of Management, Japanese Red Cross Society, \\ 1-3 Shiba Daimon 1-Chome, Minato-ku, Tokyo 105-8521, Japan
}

(Received December 12, 2016; accepted May 18, 2017)

Keywords: amorphous carbon, fluorine doping, biomaterial, antithrombogenic, anti-inflammation

When the surfaces of biomaterials come into contact with human blood, the contact can induce adhesion and activation of platelets and leukocytes following protein adhesion, and these complex reactions cause responses in the body including biomaterial-associated thrombosis and inflammation. Fluorine-incorporated amorphous carbon (a-C:H:F) is widely known as an antithrombogenic thin film and is regarded as a promising coating that can solve the problem of blood-contacting medical devices. However, the anti-inflammatory properties of a-C:H:F have not yet been elucidated. Polymorphonuclear neutrophil leukocytes (neutrophils) play important roles in thrombosis and inflammation, and platelets that adhere to and become activated on biomaterial, which are also key factors of thrombus formation, promote adhesion of neutrophils in an inflammatory process. In this study, to evaluate the antithrombogenic and anti-inflammatory properties of a-C:H:F coating, we analyzed the platelets that had adhered to and become activated on, as well as neutrophils that had adhered to, a-C:H:F-coated SUS316L, which is a conventional material used for medical devices. The a-C:H:F-coated SUS316L suppressed platelet adhesion and activation and neutrophil adhesion to a greater extent than uncoated SUS316L. These results showed that a-C:H:F coating is a suitable and biocompatible coating for implanted devices because it controls the initial thrombotic and inflammatory reactions of biomaterials.

\footnotetext{
*Corresponding author: e-mail: hasebe@tokai-u.jp

$\dagger$ These authors contributed equally to this work.

http://dx.doi.org/10.18494/SAM.2017.1558
} 


\section{Introduction}

The development of material science has taken place at medical sites, resulting in making use of powerful clinical applications. ${ }^{(1)}$ Biomaterial-based devices for blood vessels, such as in cases of catheter intervention or implantation, have gained widespread use in modern medical care, and blood-contacting material has been a particular focus of attention in biomaterial science. In accordance with the increased use of materials that come into contact with blood, host response and foreign body reaction to biomaterials remain matters of especially serious concern. Our bodies detect not only infections by viruses and bacteria but also invasion by foreign materials. When our sensitive living bodies come into contact with foreign materials, reactions such as inflammatory responses occur, regardless of biocompatibility. ${ }^{(2,3)}$ In particular, devices that come into contact with blood such as stents, vascular grafts, and artificial organs frequently induce blood thrombotic responses, as they are not completely inert in relation to blood. ${ }^{(4)}$ When coming into contact with human blood, the surfaces of biomaterials induce adhesion and activation of platelets and leukocytes following protein adhesion, and these complex reactions cause the biomaterialassociated thrombosis. ${ }^{(5-7)}$ Furthermore, adhesion and activation of leukocytes also play roles in inflammatory responses to biomaterials. ${ }^{(8)}$ Polymorphonuclear neutrophil leukocytes (neutrophils), accounting for $50-70 \%$ of leukocytes, are especially abundant in relation to thrombotic and inflammatory responses. ${ }^{(9)}$ Furthermore, activated platelets stimulate neutrophils, which affect the course of thrombosis and inflammation. ${ }^{(10-12)}$

The host response to implanted biomedical materials simultaneously involves varying degrees and stages of thrombosis and inflammation, which can lead to device failure. Therefore, the design of inert biomaterials that potentially minimize harmful responses caused by platelet and leukocyte reactions is important for blood-contacting medical device development.

Biomaterial surface properties play an important role in controlling host responses such as thrombus formation after the implantation of medical devices in blood vessels, although many factors are involved and affect the situation intricately. ${ }^{(13)}$ To improve the interaction between blood and materials, modification of surface properties is very effective, and surface coating is one way of enhancing the chemical and physical properties of materials and of reducing stimulus to biological cells. Diamond-like carbon (DLC) coating materials, such as hydrogenated amorphous carbon $(\mathrm{a}-\mathrm{C}: \mathrm{H})$ coating materials, have received much attention in this regard. These films have excellent properties such as wear resistance, high degrees of hardness, chemical inertness, corrosion resistance, and biocompatibility. ${ }^{(14,15)}$ We previously showed that fluorine doping in a-C:H films contributes to a reduction of blood cell adhesion and adsorption of plasma protein. ${ }^{(16,17)}$ We also reported that a-C:H:F-coated biomaterials can play a nontoxic mediating role in vivo, ${ }^{(18)}$ and we developed fluorine-incorporated a-C:H (a-C:H:F) coating techniques for vascular interventional devices using radio frequency plasma-enhanced chemical vapor deposition (RF-PECVD). ${ }^{(19)}$ However, the adhesion of leukocytes in relation to a-C:H:F has not yet been elucidated.

In this study, therefore, we evaluate not only the adhesion of neutrophils but also the adhesion and activation of platelets on a-C:H:F coating to verify the blood compatibility of the a-C:H:F coating. By evaluating these points for both conventional implant material stainless steel (SUS316L) and a-C:H:F-coated SUS316L, we revealed the blood compatibility of a-C:H:F film, as well as the superiority of a-C:H:F coating over bare metal. 


\section{Materials and Methods}

\section{1 a-C:H:F coating on SUS316L substrates}

The a-C:H:F film was deposited on SUS316L stainless steel disc substrates $(\varphi 15.3 \mathrm{~mm})$ from a mixture of acetylene $\left(\mathrm{C}_{2} \mathrm{H}_{2}\right.$, Koatsu Gas Kogyo Co., Ltd., Japan) and perfluoropropane $\left(\mathrm{C}_{3} \mathrm{~F}_{8}\right.$, Iwatani Corp., Japan) using RF-PECVD equipment (Onward Giken Co., Ltd., Japan). The deposition was carried out at a bias voltage of $-1.0 \mathrm{kV}$ for $10 \mathrm{~min}$, and the flow rates of $\mathrm{C}_{2} \mathrm{H}_{2}$ and $\mathrm{C}_{3} \mathrm{~F}_{8}$ gases were 10 and $100 \mathrm{sccm}$, respectively. To deposit a-C:H:F coating on SUS316L substrates without the risk of adhesion failure or cracking, silicon-incorporated a-C:H (a-C:H:Si) and hydrogenated amorphous silicon carbide (a-SiC:H) coatings were first deposited on substrates as interlayers before the deposition of the a-C:H:F film. Several studies reported that the introduction of Si-containing interlayers or multilayer coatings improved the adhesion of amorphous-carbon-based coatings to metallic substrates. ${ }^{(20,21)}$ Hasebe et al. revealed that the a-C:H:Si/a-SiC:H multi-interlayer decreased the extent of cracking and delamination between the a-C:H:F film and SUS316L stents. ${ }^{(19)}$ The deposition of a-SiC:H film was carried out for 10 min at a tetramethylsilane (TMS, Shin-Etsu Chemical Co., Ltd., Japan) flow rate of $16 \mathrm{sccm}$ at a bias voltage of $-2.0 \mathrm{kV}$ for the first interlayer. Subsequently, the deposition of a-C:H:Si film was carried out for $10 \mathrm{~min}$ at a bias voltage of $-2.0 \mathrm{kV}$ from a mixture of TMS and $\mathrm{C}_{2} \mathrm{H}_{2}$ at flow rates of 4 and $65 \mathrm{sccm}$, respectively. The thicknesses of a-C:H:F, a-C:H:Si, and a-SiC:H films from top to bottom were about 200,100 , and $100 \mathrm{~nm}$, respectively.

\subsection{Film characterization by X-ray photoelectron spectroscopy (XPS)}

The surface chemical compositions and bonding states of a-C:H:F films on the topmost surface of three-layer-coated SUS316L substrates were analyzed via XPS (K-Alpha, Thermo Fisher Scientific Inc., USA). The X-ray photoelectrons of C1s and F1s spectra were measured to confirm the existence of a-C:H:F films on coated samples. The carbon and fluorine concentrations of the film surface were calculated on the basis of peak area and corresponding relative sensitivity factors.

\subsection{Antithrombogenic test using human platelets}

\subsubsection{Pretreatment of samples}

Before experiments using human cells, each sample placed in a 24-well plate was rinsed 3 times with both $95 \%$ ethanol and phosphate-buffered saline (PBS). The samples were incubated in $2 \mathrm{ml}$ of Rosewell Park Memorial Institute (RPMI)-1640 medium (Sigma-Aldrich Co. LLC.) containing $0.1 \%$ bovine serum albumin (BSA) to prevent nonspecific binding.

\subsubsection{Preparation of human platelets and platelet culture on samples}

Human whole blood was gathered from healthy volunteers who had not ingested any medication for at least 10 days. The blood was mixed with acid-citrate-dextrose (ACD) at a ratio of 7 to 1 , and then platelet-rich plasma (PRP) was isolated by centrifugation of the blood at $1400 \mathrm{rpm}$ for 10 
min. Subsequently, residual blood was additionally centrifuged at $3000 \mathrm{rpm}$ for $15 \mathrm{~min}$ to obtain platelet-poor plasma (PPP). By diluting PRP with PPP, the density of platelets in PRP was adjusted to $3.0 \times 10^{5}$ cells $/ \mu 1$. After the adjusted PRP was left to stand for $30 \mathrm{~min}$ at room temperature, each pretreated sample with $1 \mathrm{ml}$ of adjusted PRP was incubated at $37^{\circ} \mathrm{C}$ for 60 min under shear conditions of $60 \mathrm{rpm}$. Thereafter, the PRP supernatants were removed and samples were washed with PBS to observe the adherent platelets on the surface of the samples, and supernatant PRP was removed for flow cytometry analysis.

\subsubsection{Determination of number of adherent platelets}

Platelets adhering to samples were fixed with $0.8 \mathrm{ml}$ of $1 \%$ glutaraldehyde for $12 \mathrm{~h}$ at $4{ }^{\circ} \mathrm{C}$. After fixing, they were dehydrated with a graded ethanol series $(20,40,60,80,90,95,100$, and $100 \%$ for $15 \mathrm{~min}$ each) and naturally dried in air ambient. The dried samples were observed with an optical microscope. The number of adherent platelets was determined through manual counting using photographs $\left(600 \times 440 \mu \mathrm{m}^{2}\right)$ taken from 17 randomly chosen fields per sample.

\subsubsection{Analysis of activated platelets with flow cytometry}

CD62P, known as P-selectin, platelet-activation-dependent granule external membrane (PADGEM) protein, and granule membrane protein (GMP)-140 redistribute from granule membranes to plasma membranes when platelets are activated. In addition, CD62P expression is the platelet activation marker most closely associated with increased thrombotic risk. ${ }^{(22,23)}$ P-selectin promotes fibrin deposition, inducing leukocyte accumulation, in areas of vascular injury and arterial thrombosis. ${ }^{(24,25)}$ Furthermore, the binding of P-selectin to PSGL-1 on leukocytes mediates the rolling of leukocytes on adherent and activated platelets. ${ }^{(26,27)}$

In this study, CD62P surface expression was evaluated by flow cytometry. Platelets in PRP solution were exposed to SUS316L discs and a-C:H:F coating on the discs under the conditions described in Sect. 2.3.2. The supernatants removed after incubation were immediately fixed with $4 \%$ paraformaldehyde for $15 \mathrm{~min}$ at room temperature. After fixation, the samples were adequately washed with PBS and resuspended in PBS containing $0.1 \%$ BSA and $0.01 \%$ sodium azide $\left(\mathrm{NaN}_{3}\right)$. Then, the suspension of platelets was incubated with a fluorescent-labeled monoclonal antibody to $\mathrm{CD} 62 \mathrm{P}$ for $30 \mathrm{~min}$ at $4{ }^{\circ} \mathrm{C}$ in the dark. Thereafter, the samples were washed with PBS and resuspended in $300 \mu \mathrm{l}$ of PBS containing $0.2 \% \mathrm{BSA}$ and $0.01 \% \mathrm{NaN}_{3}$, and they were then analyzed with a flow cytometer. The isotype-matched mouse IgG labeled with the same fluorescent compound was always used as a negative control. Data were analyzed using Flowjo (Tree Star, Inc., USA).

\subsection{Leukocyte adhesion}

\subsubsection{Preparation of human leukocytes and leukocyte culture on samples}

Leukocytes were isolated by the centrifugation of human whole blood with Mono-Poly Resolving Medium (DS Pharma Biomedical Co., Ltd., Japan). The blood was collected from healthy volunteers, and then mixed with ethylenediaminetetraacetic acid (EDTA). EDTAanticoagulated whole blood $(24 \mathrm{ml})$ was carefully layered on top of a mono-poly solution $(21 \mathrm{ml})$ and centrifuged at room temperature at $400 \mathrm{~g}$ for $25 \mathrm{~min}$ to separate leukocytes. After each layer 
of neutrophils, mononuclear cells and lymphocytes were carefully collected (into $50 \mathrm{~mL}$ conical centrifuge tubes). Each solution was mixed with PBS and centrifuged at $250 \mathrm{~g}$ for $10 \mathrm{~min}$ at room temperature. The isolated leukocytes (mononuclear cells and neutrophils) were suspended in $0.1 \%$ BSA-RPMI-1640 to give a final cell density of $3.0 \times 10^{6}$ cells $/ \mathrm{ml}$. For the adhesion test with neutrophils, an isolated leukocyte solution was introduced onto a-C:H:F-coated and uncoated SUS316L discs pretreated under the conditions described in Sect. 2.3.1. Mononuclear and neutrophil cells were allowed to adhere for $60 \mathrm{~min}$ at $37^{\circ} \mathrm{C}$ under shear conditions (60 rpm).

\subsubsection{Determination of number of neutrophils}

After the samples were washed with PBS following the removal of the leukocyte solutions, mononuclear cells and neutrophils adhering to the samples were fixed with $0.8 \mathrm{ml}$ of $1 \%$ glutaraldehyde for $12 \mathrm{~h}$ at $4{ }^{\circ} \mathrm{C}$. After fixing, they were dehydrated, and only adherent neutrophils were counted as described in Sect. 2.3.3.

\subsection{Statistical analysis}

The data obtained were reported as means $\pm \mathrm{SD}$, and the differences were analyzed using statistical software (JMP6.0; SAS Institute Japan Ltd., Japan). For statistical analysis of the data, Mann-Whitney's U test or the paired t-test, as well as the Wilcoxon signed-rank test, were used. A $p$-value $<0.05$ was considered to constitute a statistically significant difference.

\section{Results and Discussion}

\subsection{Film characterization}

Figure 1 shows C1s spectra of the a-C:H:F coating surface on SUS316L discs. C1s spectra showed four main peaks: C-C ( 284.4 eV), C-CF ( 287.0), C-F ( 289.0), and C-F $2(\sim 292.0)$ bonds, and the element concentrations of the a-C:H:F coating were 60.7 at.\% carbon and 39.3 at.\% fluorine. We previously reported that an a-C:H:F film with sufficient $\mathrm{C}-\mathrm{F}$ bonds on its surface showed excellent antithrombotic properties owing to its hydrophobicity. ${ }^{(16,17)}$ This spectrum led us to expect the improvement of antithrombotic properties, because it is characteristic of an antithrombotic a-C:H:F film. ${ }^{(28)}$

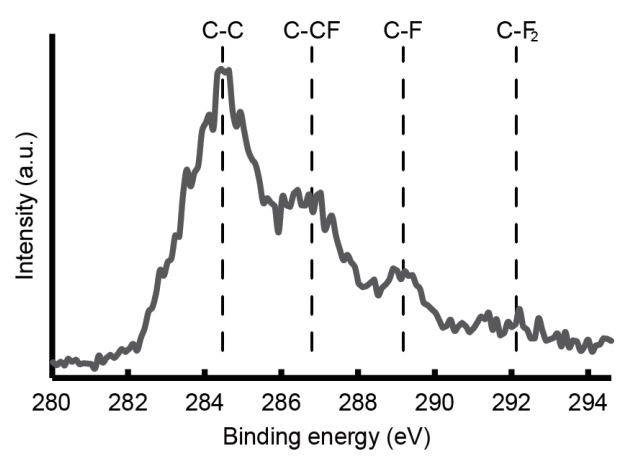

Fig. 1. C1s spectra of a-C:H:F-coated SUS316L. 


\subsection{Antithrombogenic properties}

\subsubsection{Platelet adhesion}

Figure 2 shows the number of adherent platelets on the surfaces of SUS316L discs and those coated with a-C:H:F (A, B, and C are results from 3 volunteers, and Ave. is the average value thereof). Compared with uncoated samples, the number of adherent platelets on the surface of a-C:H:F-coated samples was significantly lower, regardless of volunteer (Fig. 2, Mann-Whitney's U-test: $p<0.001$ ).

Platelets play a pivotal role in the thrombotic properties of biomaterial applications involving contact with blood. Platelet adhesion to artificial surfaces of blood-contacting devices is one of the earliest events in thrombus formation leading to platelet aggregation and activation. Therefore, the reduction of adherent platelets is a determinant factor for the eventual success of any application. In this study, a-C:H:F coating effectively suppressed platelet adhesion on SUS316L discs, regardless of volunteer. The sufficient $\mathrm{C}-\mathrm{F}$ bonds of a-C:H:F provide low surface free energy to suppress the adhesion of other substances. In a previous study from our group, we similarly reported that a-C:H:F-coated SUS suppressed adhesion of platelets on the basis of in vivo tests. ${ }^{(18)}$ It has been reported that a-C:H:F coating has sustainable antithrombogenic properties, ${ }^{(29)}$ and therefore that the improvement of SUS316L through antithrombogenic a-C:H:F coating is expected.

\subsubsection{Platelet activation}

In this study, the surface expression of $\mathrm{CD} 62 \mathrm{P}$ on platelets was measured as a marker of platelet activation. The expression of CD62P on platelets exposed to a-C:H:F-coated and uncoated SUS discs is shown in Fig. 3(a). The expression of CD62P on platelets exposed to "Uncoated SUS316L" discs was significantly higher than that in the cases involving exposure to "a-C:H:F-coated SUS316L" discs (Wilcoxon signed-rank test: $p<0.01$ ). Figure 3(b) shows the CD62P expression on platelets exposed to a-C:H:F-coated and noncoated SUS discs. In this histogram, more platelets are shown on SUS316L than on a-C:H:F-coated SUS316L discs at higher fluorescence, which means more activity. In all cases, the expression of CD62P was greater for platelets exposed to SUS316L discs than for those exposed to a-C:H:F-coated discs.

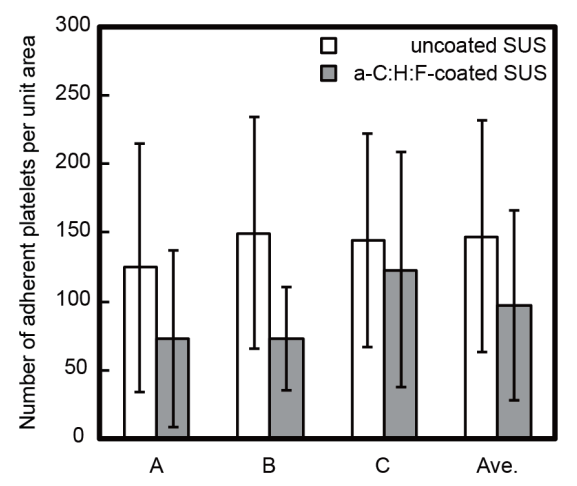

Fig. 2. Number of platelets adhering to uncoated SUS316L and a-C:H:F-coated SUS316L discs $(N=3)$. 


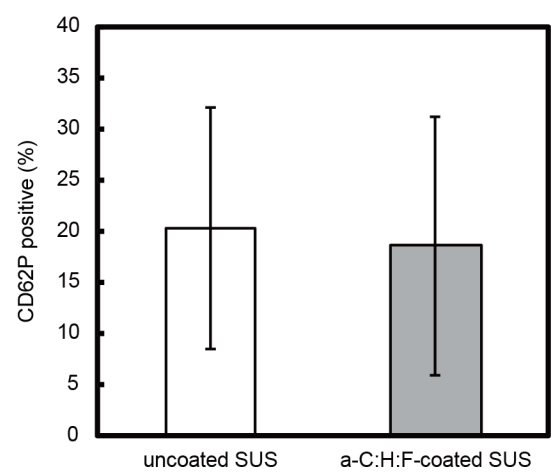

(a)

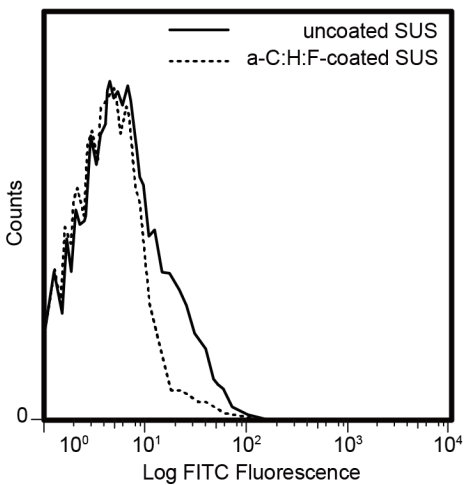

(b)

Fig. 3. (a) Number of CD62P positive platelets $(N=8)$ and (b) histogram plot of CD62P expression on platelets analyzed by flow cytometry.

In our experimental setting, we observed that platelets exposed to SUS316L discs expressed P-selectin on their surfaces, whereas such expression was significantly reduced on platelets exposed to a-C:H:F-coated SUS316L discs. These results suggested that a-C:H:F coating on SUS316L suppressed platelet activation. It is considered that the decrease in platelet adhesion on a-C:H:F coating results in the suppression of platelet activation because platelet activation is involved in platelet adhesion. ${ }^{(30)}$ These results also imply the inhibition of leukocyte accumulation, rolling, adhesion, and transmigration through interaction with activated platelets by the a-C:H:F coating. On the basis of these facts, it can be discerned that a-C:H:F coating may be effective in preventing thrombus formation and acute inflammation.

\subsection{Non-inflammatory properties}

The adsorption of neutrophils as an inflammatory marker was analyzed. Adherent neutrophil images are shown in Fig. 4, and the number of adherent neutrophils is shown in Fig. 5. Similarly to the cases of platelets, the number of granulocytes adhering to the surface of SUS316L discs coated with a-C:H:F significantly decreased compared with uncoated SUS316L disc surfaces. The number of adherent granulocytes was significantly lower on a-C:H:F-coated SUS316L discs than on uncoated SUS316L discs (Mann-Whitney's U test: $p<0.001$ ).

Neutrophils are key agents inducing various inflammatory actions, although they play important roles in host response against infectious agents. ${ }^{(10)}$ Thus, neutrophil adhesion is an important marker for the anti-inflammatory action of biomaterials, and we observed neutrophil adhesion on a-C:H:F coating. The a-C:H:F coating significantly lowered the number of adhered neutrophils compared with SUS316L discs. Neumann et al. reported that neutrophils from human whole blood or in BSA solution adhered less to the low surface tension (surface free energy) materials such as fluorocarbon resins. ${ }^{(31)}$ As a-C:H:F coating has a surface free energy lower than 30 $\mathrm{mJ} / \mathrm{m}^{2}{ }^{2}$, ${ }^{(9)}$ the adherence of neutrophils was suppressed in this study. These results demonstrated the anti-inflammatory properties of a-C:H:F coating, showing that a-C:H:F coating can reduce inflammatory risks associated with the implantation of SUS material. 


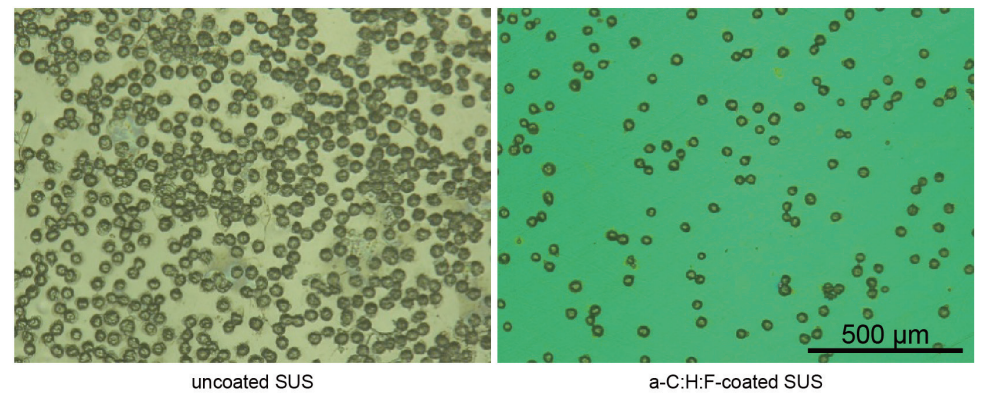

Fig. 4. (Color online) Representative pictures of granulocytes adhering to the uncoated SUS16L discs and a-C:H:F-coated SUS316L discs.

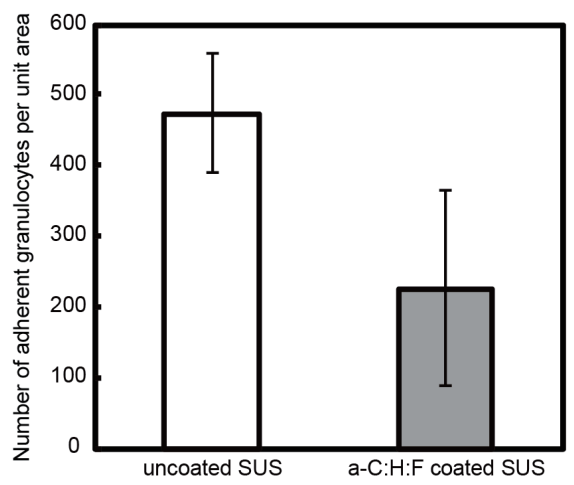

Fig. 5. Mean number of granulocytes adhering to uncoated SUS316L and a-C:H:F-coated discs $(N=3)$.

\section{Conclusions}

In this study, we evaluated the anti-thrombotic and anti-inflammatory properties of a-C:H:F film compared with SUS316L, which is used for conventional implanted devices. The a-C:H:Fcoated SUS316L suppressed platelet adhesion and activation, as well as neutrophil adhesion. These results show that a-C:H:F coating is a suitable biocompatible coating for implanted devices because it controls the initial thrombotic and inflammatory reactions of biomaterials.

\section{Acknowledgments}

This work was supported by the Nanotech Challenge Program research grant of the Research and Development Organization (NEDO) in Japan and funded partly by Kawasumi Laboratories, Inc. We greatly appreciate the discussions with and helpful comments made by Haruko Ogawa of the Graduate School of Humanities and Science and the Glycoscience Institute, Ochanomizu University; Hitoshi Okazaki, Mika Matsuhashi, and Madoka Nishimori of the Department of Transfusion Medicine and the Institute of Medical Science of the University of Tokyo; and Yoshiharu Yoshikawa, Akira Ihara, Toshiyasu Yuba, Mai Teranishi, Kazunori Murakami, and Akira Bonkohara of Kawasumi Laboratories, Inc. 


\section{References}

1 G. Binyamin, B. M. Shafi, and C. M. Mery: Semi. Pediatr. Surg. 15 (2006) 276.

2 J. M. Anderson, A. Rodriguez, and D. T. Chang: Semi. Immunol. 20 (2008) 86.

3 J. M. Anderson: Annu. Rev. Mater. Res. 31 (2001) 81.

4 S. R. Hanson: Cardiovasc. Pathol. 2 (1993) S157.

5 M. B. Gorbet and M. V. Sefton: Biomaterials 25 (2004) 5681.

6 C. D. Forbes and C. R. M. Prentice: Br. Med. Bull. 34 (1978) 201.

7 C. J. Wilson, R. E. Clegg, D. I. Leavesley, and M. J. Pearcy: Tissue Eng. 11 (2005) 1.

8 L. Tang and J. W. Eaton: Am. J. Clin. Pathol. 103 (1995) 466.

9 J. Savill, A. Wyllie, J. Henson, M. Walport, P. Henson, and C. Haslett: J. Clin. Invest. 83 (1989) 865.

10 J. A. Smith: J. Leukocyte Biol. 56 (1994) 672.

11 A. Zarbock, R. K. Polanowska-Grabowska, and K. Ley: Blood Rev. 21 (2007) 99.

12 C. S. Crovello, B. C. Furie, and B. Furie: J. Biol. Chem. 268 (1993) 14590.

13 D. F. Williams: Biomaterials 29 (2008) 2941.

14 R. Hauert: Diamond Relat. Mater. 12 (2003) 583.

15 R. K. Roy and K. R. Lee: J. Biomed. Mater. Res. B 83 (2007) 72.

16 T. Saito, T. Hasebe, S. Yohena, Y. Matsuoka, A. Kamijo, K. Takahashi, and T. Suzuki: Diamond Relat. Mater. 14 (2005) 1116.

17 T. Hasebe, S. Yohena, A. Kamijo, Y. Okazaki, A. Hotta, K. Takahashi, and T. Suzuki: J. Biomed. Mater. Res. A 83 (2007) 1192.

18 T. Hasebe, A. Shimada, T. Suzuki, Y. Matsuoka, T. Saito, S. Yohena, A. Kamijo, N. Shiraga, M. Higuchi, K. Kimura, H. Yoshimura, and S. Kuribayashi: J. Biomed. Mater. Res. A 76 (2006) 86.

19 T. Hasebe, K. Murakami, S. Nagashima, Y. Yoshimoto, A. Ihara, M. Otake, R. Kasai, S. Kasuya, N. Kitamura, A. Kamijo, H. Terada, A. Hotta, K. Takahashi, and T. Suzuki: Diamond Relat. Mater. 20 (2011) 902.

20 P. D. Maguire, J. A. McLaughlin, T. I. T. Okpalugo, P. Lemoine, P. Papakonstantinou, E. T. McAdams, M. Needham, A. A. Ogwu, M. Ball, and G. A. Abbas: Diamond Relat. Mater. 14 (2005) 1277.

21 C. Schwarz, J. Heeg, M. Rosenberg, and M. Wienecke: Diamond Relat. Mater. 17 (2008) 1685.

22 T. Inoue, Y. Sakai, T. Fujito, K. Hoshi, T. Hayashi, K. Takayanagi, S. Morooka, and R. Sohma: Circulation 94 (1996) 1523

23 D. Tschoepe, H. P. Schultheiss, P. Kolarov, B. Schwippert, K. Dannehl, H. K. Nieuwenhuis, B. Kehrel, B. Strauer, and F. A. Gries: Circulation 88 (1993) 37.

24 T. Palabrica, R. Lobb, B. C. Furie, M. Aronovitz, C. Benjamin, Y. M. Hsu, S. A. Sajer, and B. Furie: Nature 359 (1992) 848.

25 S. Yokoyama, H. Ikeda, N. Haramaki, H. Yasukawa, T. Murohara, and T. Imaizumi: J. Am. Coll. Cardiol. 45 (2005) 1280.

26 E. Larsen, A. Celi, G. E. Gilbert, B. C. Furie, J. K. Erban, R. Bonfanti, D. D. Wagner, and B. Furie: Cell 59 (1989) 305.

27 S. Hamburger and R. McEver: Blood 75 (1990) 550.

28 T. Hasebe, S. Nagashima, A. Kamijo, T. Yoshimura, T. Ishimaru, Y. Yoshimoto, S. Yohena, H. Kodama, A. Hotta, K. Takahashi, and T. Suzuki: Thin Solid Films 516 (2007) 299.

29 S. Maegawa, T. Hasebe, Y. Yamato, K. Bito, S. Nagashima, T. Hayashi, T. Mine, T. Matsumoto, A. Hotta, and T. Suzuki: Diamond Relat. Mater. 70 (2016) 33.

30 S. Kamath, A. Blann, and G. Lip: Eur. Heart J. 22 (2001) 1561.

31 A. W. Neumann, D. R. Absolom, C. J. van Oss, and W. Zingg: Cell Biophys. 1 (1979) 79. 